\title{
PICARD SETS OF ENTIRE AND MEROMORPHIC FUNCTIONS
}

\section{J. M. ANDERSON and J. CLUNIE}

\section{Introduction}

Suppose that $S$ is a subset of the complex plane $C$. We call $S$ a Picard set for entire functions if every transcendental entire function $f(z)$ assumes every complex value with at most one exception, depending on $f(z)$, infinitely often in $C \backslash S$. Similarly $S$ is a Picard set for meromorphic functions if every transcendental meromorphic function assumes every complex value infinitely often, with at most two exceptions, in $C \backslash S$. As we shall see below, Picard sets for meromorphic functions are much rarer than Picard sets for entire functions. The classical theorem of Picard asserts that any finite set of points is a Picard set for entire or meromorphic functions. Many other authors have subsequently investigated Picard sets - we refer to [6] for details. The starting point of this investigation is the following theorem of Baker and Liverpool ([1], Theorem 2).

Theorem A. Suppose that $q>1$ is given. Then there is a constant $K=K(q)$ such that, for every complex sequence $\left\{a_{n}\right\}$ satisfying

$$
\left|\frac{a_{n+1}}{a_{n}}\right|>q>1, \quad n=1,2,3, \ldots,
$$

and every positive sequence $\left\{\varrho_{n}\right\}$ satisfying

$$
\log \frac{1}{\varrho_{n}}>K \frac{\left(\log \left|a_{n}\right|\right)^{2}}{\log q}, \quad n>n_{0} \quad \text { say, }
$$

the set

$$
S=\bigcup_{n=1}^{\infty} D_{n}, \text { where } D_{n}=\left\{z:\left|z-a_{n}\right|<\varrho_{n}\right\}
$$

is a Picard set for entire functions.

The value given by Baker and Liverpool for $K$ is $4(q+1) /(q-1)$, and they show by an example that $K<1 / 2$ is, in general, not sufficient. Here we shall show that any $K>1 / 2$ is sufficient. This result is then more or less best possible with the critical case $K=1 / 2$ left undecided. To be precise we shall show 
Theorem 1. Suppose that $\left\{a_{n}\right\}$ is a complex sequence and $\left\{\varrho_{n}\right\}$ a positive sequence satisfying (1.1) and (1.2) for some $K>1 / 2$. Then $S$, defined by (1.3), is a Picard set for entire functions.

No such similar result holds in general for meromorphic functions. This follows, as has been pointed out by Toppila ([4], p. 10), from a consideration of the function

$$
F(z)=\prod_{n=1}^{\infty}\left(\frac{z-a_{n}}{z-a_{n}-\delta_{n}}\right)
$$

for suitable positive sequences $\left\{a_{n}\right\}$ and $\left\{\delta_{n}\right\}$. We may choose the sequence $\left\{a_{n}\right\}$ to satisfy (1.1) and, given any sequence $\left\{\varrho_{n}\right\}_{\downarrow} 0$ we may choose the $\delta_{n}$ 's so small that outside the discs $D_{n}=\left\{z:\left|z-a_{n}\right|<\varrho_{n}\right\}$ we have

$$
\frac{1}{K} \leqq|F(z)| \leqq K
$$

for some absolute constant $K$. Thus $S=\cup_{n=1}^{\infty} D_{n}$ is not a Picard set for meromorphic functions. However, such functions $F(z)$ do not have any Nevanlinna deficient values. This turns out to play a critical role and our arguments can be adapted, with some loss of precision, to deal with the case of meromorphic functions having a deficient value. We prefer to prove Theorem 1 first, to illustrate the method, and then discuss the generalizations in Sections 3 and 4.

\section{Proof of Theorem 1}

In what follows we assume acquaintance with the standard terminology of the Nevanlinna theory, as given in [3], and we use it without further introduction. Suppose that $S$, defined by (1.3), is not a Picard set so that there is a transcendental entire function $f(z)$ such that for some $a, b \in C, a \neq b$, all the $a$-points and $b$-points of $f(z)$, apart, perhaps, from a finite number, lie in $S$. We assume, without loss of generality, that $a=0, b=1$. An elementary argument involving Schottky's Theorem (see e.g. [1], p. 232) shows that, if $A_{n}$ denotes the annulus $\left\{z:\left|a_{n}\right| q^{1 / 3} \leqq|z| \leqq\left|a_{n}\right| q^{2 / 3}\right\}$, $n=1,2,3, \ldots$, then $|f(z)| \rightarrow \infty$ as $z \rightarrow \infty, z \in A_{n}$, uniformly with respect to $\theta=\arg z$. Thus $f(z)$ has no finite deficient values and so 0 and 1 are assumed infinitely often. Since the variations of $\arg f(z)$ and $\arg (f(z)-1)$ as we describe the circles $|z|=$ $\left|a_{n}\right| q^{1 / 2}, n=1,2,3, \ldots$ are equal, we see from the Argument Principle that the number of zeros and 1-points in $D_{n}$ must be the same, for all large $n$. We denote this number by $v_{n}$. There are two cases to consider.

Case 1.

$$
\limsup _{n \rightarrow \infty} v_{n}=N<\infty \text {. }
$$


In this case we have $N \geqq 1$ and $v_{n} \leqq N$ for all large $n$ with $v_{n}=N$ infinitely often. If $k \in N$ and $t$ satisfies $q^{k-1}\left|a_{1}\right|<t \leqq q^{k}\left|a_{1}\right|$ then the number of $a_{n}$ 's not exceeding $t$ is at most $k$. Hence, as $t \rightarrow \infty$

$$
\begin{aligned}
n(t, 0) & \leqq N k+O(1) \\
& \leqq(1+o(1)) N \frac{\log t}{\log q} .
\end{aligned}
$$

A similar estimate is true for $n(t, 1)$ and so we deduce that

$$
\begin{gathered}
N(r, 0) \leqq(1+o(1)) N \frac{(\log r)^{2}}{2 \log q}, \\
N(r, 1) \leqq(1+o(1)) N \frac{(\log r)^{2}}{2 \log q} \quad(r \rightarrow \infty) .
\end{gathered}
$$

It now follows from Nevanlinna's second fundamental theorem ([3], p. 43), or indeed, from more elementary considerations, that

$$
T(r, f)=O(\log r)^{2} \quad(r \rightarrow \infty) .
$$

Hence, by a known result on functions of slow growth ([5]),

and so, in particular

$$
\log M(r, f) \sim N(r, 0) \quad(r \rightarrow \infty),
$$

$$
\log M(r, f) \leqq(1+o(1)) N \frac{(\log r)^{2}}{2 \log q}(r \rightarrow \infty) .
$$

We now consider large $n$ with $v_{n}=N$ and denote the zeros of $f$ in $L_{n}$ by $\zeta_{1}, \ldots, \zeta_{N}$. We define

$$
g(z)=f(z) \prod_{k=1}^{N}\left(z-\zeta_{k}\right)^{-1}
$$

On the circle $\left|z-a_{n}\right|=1$ we have the obvious estimate

$$
|g(z)| \leqq \frac{|f(z)|}{\left(1-\varrho_{n}\right)^{N}} \leqq \exp \left\{(1+o(1)) N \frac{\left(\log \left(\left|a_{n}\right|+1\right)\right)^{2}}{2 \log q}\right\}\left(1-\varrho_{n}\right)^{-N} .
$$

Hence, for $\left|z-a_{n}\right| \leqq 1$ we obtain

$$
|f(z)| \leqq\left(\prod_{k=1}^{N}\left|z-\zeta_{k}\right|\right)\left(1-\varrho_{n}\right)^{-N} \exp \left\{(1+o(1)) \frac{N\left(\log \left|a_{n}\right|\right)^{2}}{2 \log q}\right\} .
$$

If we now choose $z \in D_{n}$ so that $f(z)=1$ we obtain

$$
(1+o(1)) N \frac{\left(\log \left|a_{n}\right|\right)^{2}}{2 \log q}+N \log \left(\frac{2 \varrho_{n}}{1-\varrho_{n}}\right) \geqq 0 .
$$


Since we may assume $n$ sufficiently large so that $\varrho_{n}<1 / 2$ we obtain, finally, on dividing by $N \neq 0$,

$$
\log \frac{1}{\varrho_{n}} \leqq(1+o(1)) \frac{\left(\log \left|a_{n}\right|\right)^{2}}{2 \log q},
$$

which contradicts (1.2) with $K>1 / 2$.

Case II.

$$
\limsup _{n \rightarrow \infty} v_{n}=\infty .
$$

This is the more difficult case since here the growth of $f(z)$ may not be slow and so the reasoning is somewhat different from that above. We define

$$
\mu_{n}=\max v_{k} \quad(k<n)
$$

and consider again two sub-cases.

Case IIa.

$$
\liminf _{n \rightarrow \infty} \frac{\mu_{n}}{v_{n}}=0 .
$$

We pass to a subsequence $\sigma$ of $N$ such that $\mu_{n}=o\left(v_{n}\right)(n \rightarrow \infty, n \in \sigma)$, and we consider only such values of $n$. As before, for $q^{k-1}\left|a_{1}\right|<t \leqq q^{k}\left|a_{1}\right|$, the number of $a_{v}$ 's not exceeding $t$ is at most $k$. We set $r_{n}=q^{1 / 2}\left|a_{n}\right|$ and then, for $|t| \leqq r_{n}$ we have

$$
n(t, 0) \leqq \begin{cases}\mu_{n} k+O(1), & t \leqq\left|a_{n}\right|-\varrho_{n}, \\ \mu_{n} k+v_{n}+O(1), & \left|a_{n}\right|-\varrho_{n}<t \leqq r_{n}=q^{1 / 2}\left|a_{n}\right| .\end{cases}
$$

Since $k=(1+o(1)) \log t / \log q(t \rightarrow \infty)$ we find, on integrating and making some obvious estimates that

$$
\begin{gathered}
N\left(r_{n}, 0\right) \leqq(1+o(1)) \mu_{n} \frac{\left(\log r_{n}\right)^{2}}{2 \log q}+v_{n} \log \frac{r_{n}}{\left|a_{n}\right|-\varrho_{n}} \\
\leqq(1+o(1))\left\{\mu_{n} \frac{\left(\log \left|a_{n}\right|\right)^{2}}{2 \log q}+\frac{v_{n}}{2} \log q\right\} .
\end{gathered}
$$

But, as we have shown previously, $f(z) \rightarrow \infty$ uniformly in $\theta$ as $z=r_{n} e^{i \theta} \rightarrow \infty$. Hence

$$
T\left(r_{n}, f\right)=N\left(r_{n}, 0\right)+O(1) \quad(n \rightarrow \infty) .
$$

Hence, by Jensen's formula ([3], Theorem 1.6) we have, for $r=|z|<\left|a_{n}\right|+1$,

$$
\begin{aligned}
& \log M(r, f) \leqq \frac{r_{n}+\left|a_{n}\right|+1}{r_{n}-\left|a_{n}\right|-1}\left\{N\left(r_{n}, 0\right)+O(1)\right\} \\
\leqq & \frac{q^{1 / 2}+1}{q^{1 / 2}-1}(1+o(1))\left\{\mu_{n} \frac{\left(\log \left|a_{n}\right|\right)^{2}}{2 \log q}+\frac{1}{2} v_{n} \log q\right\},
\end{aligned}
$$

from (2.2). We now argue in the analogous manner to Case I using the above inequality instead of (1.3). We obtain

$$
v_{n} \log \left(\frac{2 \varrho_{n}}{1-\varrho_{n}}\right)+\frac{q^{1 / 2}+1}{q^{1 / 2}-1}(1+o(1))\left\{\mu_{n} \frac{\left(\log \left|a_{n}\right|\right)^{2}}{2 \log q}+\frac{1}{2} v_{n} \log q\right\} \geqq 0
$$


which yields, for $n \in \sigma$

$$
\log \frac{1}{\varrho_{n}} \leqq \frac{q^{1 / 2}+1}{q^{1 / 2}-1}(1+o(1))\left\{\frac{\mu_{n}}{v_{n}} \frac{\left(\log \left|a_{n}\right|\right)^{2}}{2 \log q}+\frac{1}{2} \log q\right\} .
$$

Since $\mu_{n} / v_{n} \rightarrow 0$ as $n \rightarrow \infty n \in \sigma$ we see that in this case (1.2) is contradicted for any $K>0$.

$$
\text { Case IIb. } \quad \liminf _{n \rightarrow \infty} \frac{\mu_{n}}{v_{n}}>0 .
$$

Since we are assuming, in any case, that $\lim _{\sup _{n \rightarrow \infty}} v_{n}=\infty$ we consider in this case a subsequence $\sigma_{1}$ of $N$ such that $\mu_{n}<v_{n}\left(n \in \sigma_{1}\right)$. If

$$
\liminf _{n \rightarrow \infty} \frac{\mu_{n}}{v_{n}}>\frac{1}{\alpha}>0 \quad(\alpha>1)
$$

then, for all $n$ sufficiently large, $v_{n+1}<\alpha v_{n}$ and, for all $k \in N, v_{n+k}<\alpha^{k} v_{n}$. The number $k$ is at our disposal, to be chosen later. With $r_{n}=q^{1 / 2}\left|a_{n}\right|$ and $R_{n}=q^{-1 / 2}\left|a_{n+1}\right|$ we estimate $N(r, 0)$ for $r_{n+k} \leqq r \leqq R_{n+k}$ noting, as before, that $T(r, f)=N(r, 0)+O(1)$ for such values of $r$. As in Case IIa we have

$$
n(t, 0) \leqq(1+o(1)) \mu_{n} \frac{\log t}{\log q} \text { for } \quad|t| \leqq\left|a_{n}\right|-\varrho_{n} .
$$

For $\left|a_{n}\right|-\varrho_{n}<t \leqq r$, where $r_{n+k} \leqq r \leqq R_{n+k}$, we have

$$
\begin{aligned}
n(t, 0) & \leqq n\left(\left|a_{n}\right|-\varrho_{n}, 0\right)+v_{n}+v_{n+1}+\ldots+v_{n+k} \\
& \leqq n\left(\left|a_{n}\right|-\varrho_{n}, 0\right)+\frac{\alpha}{\alpha-1} \alpha^{k} v_{n} .
\end{aligned}
$$

Hence, for $r_{n+k} \leqq r \leqq R_{n+k}$,

$$
N(r, 0) \leqq(1+o(1)) \mu_{n} \frac{(\log r)^{2}}{2 \log q}+K \alpha^{k} v_{n} \log \left(\frac{r}{\left|a_{n}\right|-\varrho_{n}}\right),
$$

for some suitable constant $K$.

We fix an $r_{0}$ in $\left[r_{n+k}, R_{n+k}\right]$ for which (2.3) holds and define $s$ by

$$
\left|a_{n}\right| q^{s} \leqq r_{0}<\left|a_{n}\right| q^{s+1} .
$$

Clearly $k \leqq s$ and so we obtain

$$
N\left(r_{0}, 0\right) \leqq(1+o(1)) \mu_{n} \frac{\left(\log \left|a_{n}\right|+(s+1) \log q\right)^{2}}{2 \log q}+K \exp (s \log \alpha) v_{n}(s+1) \log q
$$

We now reverse this process. For fixed $n \in \sigma$, we choose $s=\left[\left(\log \log \left|a_{n}\right|\right) /(2 \log \alpha)\right]$ and note that $s \rightarrow \infty$ as $n \rightarrow \infty$. We then choose $k=k(s)$ so that $k \leqq s$ and such that there is an $r_{0}$ satisfying simultaneously

$$
\begin{gathered}
r_{n+k} \leqq r_{0} \leqq R_{n+k}, \\
\left|a_{n}\right| q^{s} \leqq r_{0} \leqq\left|a_{n}\right| q^{s+1} .
\end{gathered}
$$


We remark that such a choice of $r_{0}$ is always possible since $R_{n+k-1}=q r_{n+k}$ for all $n$ and $k$. Thus

$$
\begin{aligned}
T\left(r_{0}, f\right) & =N\left(r_{0}, f\right)+O(1) \\
& \leqq(1+o(1)) \mu_{n} \frac{\left(\log \left|a_{n}\right|\right)^{2}}{2 \log q}+K_{1} v_{n}\left(\log \left|a_{n}\right|\right) \\
& =(1+o(1)) \mu_{n} \frac{\left(\log \left|a_{n}\right|\right)^{2}}{2 \log q}
\end{aligned}
$$

since $v_{n}<\alpha \mu_{n}$ for all sufficiently large $n$, and by our choice of $s$.

Hence, for $r=|z| \leqq\left|a_{n}\right|+1$,

$$
\begin{aligned}
\log M\left(r_{0}, f\right) & \leqq \frac{r_{0}+\left|a_{n}\right|+1}{r_{0}-\left|a_{n}\right|-1} T\left(r_{0}, f\right) \\
& \leqq \frac{q^{s}+1}{q^{s}-1}(1+o(1)) \mu_{n} \frac{\left(\log \left|a_{n}\right|\right)^{2}}{2 \log q} .
\end{aligned}
$$

We now proceed precisely as in the previous two cases to obtain

$$
v_{n} \log \left(\frac{2 \varrho_{n}}{1-\varrho_{n}}\right)+\frac{q^{s}+1}{q^{s}-1}(1+o(1)) \mu_{n} \frac{\left(\log \left|a_{n}\right|\right)^{2}}{2 \log q} \geqq 0,
$$

for all sufficiently large $n$ in $\sigma_{1}$. Since $s \rightarrow \infty$ as $n \rightarrow \infty$ we obtain

$$
\log \frac{1}{\varrho_{n}} \leqq(1+o(1)) \frac{\mu_{n}}{v_{n}} \frac{\left(\log \left|a_{n}\right|\right)^{2}}{2 \log q} .
$$

But $\mu_{n}<v_{n}$ for $n \in \sigma_{1}$ and so we arrive at a contradiction with (1.2) unless $K<1 / 2$. Thus Theorem 1 is proved in all cases.

\section{Meromorphic functions with $\delta(a, f)>0$}

Results similar to Theorem 1, but less precise, remain true for meromorphic functions with a deficient value. We suppose, without loss of generality that $\delta(\infty, f)>0$ and discuss some properties of meromorphic functions which are germane to our context. As before we assume that the sequence $\left\{a_{n}\right\}$ satisfies (1.1) and that the set $S$ is defined by (1.3). We define, for $0<\delta \leqq 1$,

$$
M(\delta)=\{f(z): f \text { meromorphic in } C, \delta(\infty, f) \geqq \delta\}
$$

and prove the following theorem. 
Theorem 2. Suppose that $q$ and $\delta$ are given with $q>1,0<\delta \leqq 1$. Then, if the sequence $\left\{a_{n}\right\}$ satisfies (1.1), there exists a constant $K=K(q)$ such that, if

$$
\log \frac{1}{\varrho_{n}}>K \delta^{-2} \log \frac{2}{\delta}\left(\log \left|a_{n}\right|\right)^{2}
$$

then $S$ defined by (1.3) is a Picard set for $M(\delta)$.

The proof again falls into two cases giving different values for the constant $K(q)$ and a different dependence on $\delta$. The question of how far these results are from being best possible is discussed in Section 5. To prove Theorem 2 we argue, as before, by contradiction and suppose that there is a transcendental meromorphic function $f(z) \in M(\delta)$ all of whose $a, b, c$-points apart from finitely many, lie in $S=\cup_{n=1}^{\infty} D_{n}$. Here $a, b$ and $c$ are three distinct values in the extended complex plane. Without loss of generality we may take $a=0, b=1$, but $c$ is not at our disposal since we have already made the assumption that $\delta(\infty, f) \geqq \delta$. Thus $c$ may equal $\infty$, or may not and we consider these two cases separately.

Case I. $c=\infty$.

In this case we have

Theorem 3. Suppose that the sequence $\left\{a_{n}\right\}$ satisfies (1.1) and that $S$ is defined by (1.3). Suppose, further, that $f(z) \in M(\delta)$ and that all the $0,1, \infty$ points of $f(z)$, apart from finitely many, lie in $S$. Then there is a $K=K(q)$ such that, if

then $f(z)$ is rational.

$$
\log \frac{1}{\varrho_{n}}>K \delta^{-2}\left(\log \left|a_{n}\right|\right)^{2} \quad\left(n \geqq n_{0}\right),
$$

Case II. $c \neq \infty$.

In this case we have

Theorem 4. Suppose that the sequence $\left\{a_{n}\right\}$ satisfies (1.1) and that $S$ is defined by (1.3). Suppose, further, that $f(z) \in M(\delta)$ and that all the 0,1,c-points of $f(z)$, apart from finitely many, lie in $S$, where $c \neq \infty$. Then there is a $K=K(q)$ such that, if

then $f(z)$ is rational.

$$
\log \frac{1}{\varrho_{n}}>K \delta^{-2} \log \frac{2}{\delta}\left(\log \left|a_{n}\right|\right)^{2} \quad\left(n \geqq n_{0}\right)
$$

Clearly Theorem 2 is an immediate consequence of Theorems 3 and 4 . The proofs are, in both cases, modifications of the proof of Theorem 1, but various complications arise. We consider firstly

Case I. $c=\infty$. 
Suppose that the transcendental meromorphic function $f(z)$ satisfies the hypotheses of Theorem 3. If $\min _{\theta}\left|f\left(r e^{i \theta}\right)\right|$ were bounded for some $r$ satisfying $\left|a_{n}\right| q^{\varepsilon} \leqq$ $r \leqq\left|a_{n}\right| q^{1-\varepsilon}$ as $n \rightarrow \infty$, where $\varepsilon>0$ is arbitrary, then it would follow, by the same application of Schottky's Theorem as in Section 2, that $\max _{\theta}\left|f\left(r e^{i \theta}\right)\right|$ would be bounded for those values of $r$ too. But this contradicts the fact that $\infty$ is a deficient value of $f(z)$, and so $f\left(r e^{i \theta}\right) \rightarrow \infty$ uniformly with respect to $\theta$ as $r \rightarrow \infty$ in these annuli. Since $f(z)$ and $f(z)-1$ have the same number of poles in the annuli $\left|a_{n}\right| q^{1 / 2} \leqq|z| \leqq$ $\left|a_{n+1}\right| q^{1 / 2}$ (in fact they are all in the disc $D_{n}$ for all sufficiently large $n$ ) an app ication of the Argument Principle again shows us that $f(z)$ has the same number of 0 's and 1's in $D_{n}$ for all $n \geqq n_{0}$, say. As before we denote this number by $v_{n}$ and note that, since $\infty$ is the only deficient value of $f(z)$, we have $v_{n} \geqq 1$ at least for a sequence of $n$ approaching infinity.

Let $p_{n}, n \geqq 1$, be the number of poles of $f(z)$ in $D_{n}$. We define

$$
E=\left\{n \in N: v_{n}>0, p_{n}<(1-\delta / 2) v_{n}\right\} .
$$

We see that $E$ must contain infinitely many elements since, otherwise, for all sufficiently large $r$,

$$
N(r, \infty, f) \geqq(1+o(1))(1-\delta / 2) N(r, 0, f) .
$$

Applying this inequality at the values $r=\left|a_{n}\right| q^{1 / 2}, n=1,2, \ldots$, where

we would obtain that

$$
N(r, 0, f)=T(r, f)+O(1),
$$

$$
\limsup _{r \rightarrow \infty} \frac{N(r, \infty)}{T(r)} \geqq 1-\delta / 2,
$$

which contradicts the fact that the deficiency of $f(z)$ is at least $\delta$.

We assume that $0 \notin \bigcup_{n \in E} D_{n}$ and introduce the notation

$$
\begin{gathered}
\hat{n}(t, 0)=\text { number of zeros of } f(z) \text { in }\{|z| \leqq t\} \cap \bigcup_{n \in E} D_{n} \\
\hat{N}(t, 0)=\int_{0}^{t} \frac{\hat{n}(u, 0)}{u} d u .
\end{gathered}
$$

The quantities $\hat{n}(t, \infty), \hat{n}(t, 1), \hat{N}(t, \infty), \hat{N}(t, 1)$ are defined similarly. Then, from the definition of $E$,

$$
\begin{gathered}
N(r, 0)-\hat{N}(r, 0) \leqq(1-\delta / 2)^{-1}[N(r, \infty)-\hat{N}(r, \infty)] \\
\leqq(1+o(1))(1-\delta)(1-\delta / 2)^{-1} T(r, f) \quad(r \rightarrow \infty) .
\end{gathered}
$$

If we apply this inequality to values $r$ for which $T(r, f)=N(r, 0)+O(1)$, in particular to values $r$ lying in annuli of the above type where $f\left(r e^{i \theta}\right) \rightarrow \infty$ as $r \rightarrow \infty$ uniformly with respect to $\theta$ we obtain

$$
\begin{aligned}
\hat{N}(r, 0) & \geqq N(r, 0)-(1+o(1))(1-\delta)(1-\delta / 2)^{-1} T(r, f) \\
& \geqq(1-o(1)) \delta(2-\delta)^{-1} T(r, f) .
\end{aligned}
$$


Similarly we have

$$
\hat{N}(r, 1) \geqq(1+o(1)) \delta(2-\delta)^{-1} T(r, f) .
$$

In particular, since $2-q^{-1 / 2}<q$ for all $q>1$, the value $r=\left(2-q^{-1 / 2}\right)\left|a_{n}\right|$ is a permissible value of $r$.

However $\log |f(z)| \leqq T(r, f)+O(1)$ for some $z$ with $|z|=r=\left(2-q^{-1 / 2}\right)\left|a_{n}\right|$. Hence, by Schottky's Theorem there is a constant $A=A(q)$ such that

$$
\log |f(z)| \leqq A T(r, f) \leqq A \delta^{-1}(2-\delta) \hat{N}(r, 0)
$$

for $|z|=r=\left(2-q^{-1 / 2}\right)\left|a_{n}\right|$. The same inequality holds, possibly with a different choice of the constant $A$ on the circle $\left|z-a_{n}\right|=\left(1-q^{-1 / 2}\right)\left|a_{n}\right|$ by applying Schottky's theorem again and noting that the circles $|z|=\left(2-q^{-1 / 2}\right)\left|a_{n}\right|$ and $\left|z-a_{n}\right|=$ $\left(1-q^{-1 / 2}\right)\left|a_{n}\right|$ have a point in common.

We remark that the dependence of $A$ on $q$ is relatively complicated and we have no reason to believe that, in the applications which follow, the estimates for $A(q)$ are best possible. For this reason we concern ourselves, in what follows, only with the dependence on $\delta$ and we will denote by $K=K(q)$ a generic constant, not necessarily the same at each occurrence.

As before, for $n \in E$ we define $\mu_{n}=\max \left\{v_{k}: k<n k \in E\right\}$. For fixed $n \in E$ we let $z_{1}, z_{2}, \ldots, z_{N}\left(N=v_{n}\right)$ and $\zeta_{1}, \zeta_{2}, \ldots, \zeta_{M}\left(M=p_{n}\right)$ be the zeros and poles of $f(z)$ in $D_{n}$ and consider

$$
g(z)=f(z) \prod_{k=1}^{M}\left(z-\zeta_{k}\right) \prod_{j=1}^{N}\left(z-z_{j}\right)^{-1}
$$

From the previous discussion it follows that, for $\left|z-a_{n}\right|=\left(1-q^{-1 / 2}\right)\left|a_{n}\right|$,

$$
\log |g(z)| \leqq \delta^{-1}(2-\delta) A \hat{N}(r, 0)+\log \left|\prod_{k=1}^{M}\left(z-\zeta_{k}\right) \prod_{j=1}^{N}\left(z-z_{j}\right)^{-1}\right|,
$$

where, as before $r=\left(2-q^{-1 / 2}\right)\left|a_{n}\right|$. Since $M<(1-\delta / 2) N$, the contribution from the last term above is negative, provided $n$ is large enough.

We now have to consider the same three cases, Case I, Case IIa and Case IIb as in Section 2, depending on the relative sizes of $\mu_{n}$ and $v_{n}$. However in all three cases we obtain, as before, with $r=\left(2-q^{-1 / 2}\right)\left|a_{n}\right|$,

$$
\hat{N}(r, 0) \leqq K(q) v_{n}\left(\log \left|a_{n}\right|\right)^{2} .
$$

Thus, for $\left|z-a_{n}\right| \leqq\left(1-q^{-1 / 2}\right)\left|a_{n}\right|$ we have, combining (3.1), (3.2) and (3.3),

$$
\log |f(z)| \leqq \log \left|\prod_{j=1}^{N}\left(z-z_{j}\right) \prod_{k=1}^{M}\left(z-\zeta_{k}\right)^{-1}\right|+K \delta^{-1} v_{n}\left(\log \left|a_{n}\right|\right)^{2} .
$$

We can argue as above with $f(z)-1$ in place of $f(z)$. If $\omega_{1}, \omega_{2}, \ldots, \omega_{N}$ are the 1-points of $f(z)$ in $D_{n}$ - there are the same number $N=v_{n}$ of such points then for $\left|z-a_{n}\right| \leqq\left(1-q^{-1 / 2}\right)\left|a_{n}\right|$ we have

$$
\log |f(z)-1| \leqq \log \left|\prod_{j=1}^{N}\left(z-\omega_{j}\right) \prod_{k=1}^{M}\left(z-\zeta_{k}\right)^{-1}\right|+K \delta^{-1} v_{n}\left(\log \left|a_{n}\right|\right)^{2}
$$


At all points on the circle $\left\{\left|z-a_{n}\right|=2 \varrho_{n}\right\}$ either $|f(z)| \geqq 1 / 2$ or $|f(z)-1| \geqq 1 / 2$, whereas $\left|z-z_{j}\right| \leqq 3 \varrho_{n}, \quad\left|z-\omega_{j}\right| \leqq 3 \varrho_{n}, \quad\left|z-\zeta_{k}\right| \geqq \varrho_{n}$.

Now pick any such point $z$ and use estimate (3.4) if $|f(z)| \geqq 1 / 2$ or (3.5) if $|f(z)-1| \geqq$ $1 / 2$ to obtain

$$
-\log 2 \leqq N \log 3+(N-M) \log \varrho_{n}+K(q) \delta^{-1} v_{n}\left(\log \left|a_{n}\right|\right)^{2} .
$$

But $N-M=v_{n}-p_{n}>\delta v_{n} / 2$ since $n \in E$. Thus

$$
v_{n} \frac{\delta}{2} \log \frac{1}{\varrho_{n}} \leqq v_{n} \log 3+K \delta^{-1} v_{n}\left(\log \left|a_{n}\right|\right)^{2} .
$$

Hence, if $E$ is not a Picard set,

and so Theorem 3 is proved.

$$
\log \frac{1}{\varrho_{n}} \leqq K(q) \delta^{-2}\left(\log \left|a_{n}\right|\right)^{2},
$$

Case II. $c \neq \infty$.

The method of proof in this case is the same as in Case I, but various technical complications arise.

Lemma 1. Suppose that $f(z)$ satisfies the hypotheses of Theorem 4. Then, for any $Q$ with $1<Q<q^{1 / 2}, f\left(r e^{i \theta}\right) \rightarrow \infty$ uniformly with respect to $\theta$ as $r \rightarrow \infty$ through the set of annuli $\cup_{n=1}^{\infty}\left\{Q\left|a_{n}\right| \leqq r \leqq Q^{-1}\left|a_{n+1}\right|\right\}$.

To avoid breaking in to the argument we postpone the proof of Lemma 1 to Section 4. Its use in the proof of Theorem 4 replaces the standard applications of Schottky's Theorem for an annulus which we have made in all the previous arguments.

We now let $v_{n}$ denote the number of zeros of $f(z)$ in $D_{n}$ and let $p_{n}$ denote the number of poles of $f(z)$ in the annulus $\left\{Q^{-1}\left|a_{n}\right| \leqq|z| \leqq Q\left|a_{n}\right|\right\}$ for some fixed $Q$, $1<Q<q^{1 / 2}$. As before we define

$$
E=\left\{n \in N: v_{n}>0, p_{n}<(1-\delta / 2) v_{n}\right\}, \quad \delta=\delta(\infty, f),
$$

and note that $E$ contains infinitely many elements. We define $\hat{n}(t, 0)$ and $\hat{N}(t, 0)$ as before.

Suppose now that $k \notin E$ and consider the annulus $\left\{Q^{-1}\left|a_{k}\right| \leqq|z| \leqq Q\left|a_{k}\right|\right\}$. The contribution of a zero or pole in this annulus to $N(R, 0)$ or $N(R, \infty)$ will differ from $\log ^{+}\left(R /\left|a_{k}\right|\right)$ by at most $\log Q$. Hence if $\alpha_{k}$ and $\beta_{k}$ are the contributions to $N(R, 0)$ and $N(R, \infty)$ from the zeros and poles in this annulus then, since $k \notin E$,

Thus

$$
\alpha_{k}-v_{k} \log Q \leqq(1-\delta / 2)^{-1}\left(\beta_{k}+p_{k} \log Q\right) .
$$

$$
\alpha_{k} \leqq(1-\delta / 2)^{-1}\left(\beta_{k}+2 p_{k} \log Q\right) \text {. }
$$


Hence, summing over all such $k \notin E$ we obtain, with $R=q^{1 / 2}\left|a_{n}\right|$,

$$
N(R, 0)-\hat{N}(R, 0) \leqq(1+o(1))(1-\delta / 2)^{-1}\left[N(R, \infty)+2 n\left(Q\left|a_{n}\right|, \infty\right) \log Q\right] .
$$

We estimate $n\left(Q\left|a_{n}\right|, \infty\right)$ by the obvious inequality

and obtain

$$
n\left(Q\left|a_{n}\right|, \infty\right) \log \frac{q^{1 / 2}}{Q} \leqq \int_{Q\left|a_{n}\right|}^{q^{1 / 2}\left|a_{n}\right|} n(t, \infty) \frac{d t}{t} \leqq N(R, \infty)
$$

$$
\begin{gathered}
N(R, 0)-\hat{N}(R, 0) \leqq(1+o(1))(1-\delta / 2)^{-1} N(R, \infty)\left\{1+2 \log Q \log \left(q^{1 / 2} / Q\right)\right\} \\
\leqq(1+o(1))(1-\delta)(1-\delta / 2)^{-1} T(R, \infty)\left\{1+2 \log Q \log \left(q^{1 / 2} / Q\right)\right\} .
\end{gathered}
$$

The quantity $Q$ is still at our disposal and we now choose

to obtain

$$
Q=\min \left(e^{1 / 2},(1+\delta / 4)^{-1} q^{1 / 2}\right)<q^{1 / 2}
$$

$$
\hat{N}(R, 0) \geqq N(R, 0)-(1+o(1))(1-\delta)(1-\delta / 2)^{-1}(1+\delta / 4) T(R, f) .
$$

But $R=\left|a_{n}\right| q^{1 / 2}$ and hence, by Lemma $1, N(R, 0)=T(R, f)+O(1)(R \rightarrow \infty)$. We finally obtain

$$
\begin{gathered}
\hat{N}(R, 0) \geqq T(R, f)\left\{1-\frac{(1-\delta)(1+\delta / 4)}{1-\delta / 2}\right\}(1+o(1)), \\
\geqq(1+o(1)) \frac{\delta}{4} T(R, f) \quad\left(R=q^{1 / 2}\left|a_{n}\right|\right) .
\end{gathered}
$$

This estimate is, in fact, valid for $R \leqq r \leqq Q^{-1}\left|a_{n+1}\right|$.

Once again we define $\mu_{n}=\max \left\{v_{k}: k<n, k \in E\right\}$ and consider the same three separate cases as in Section 2, depending on the relative sizes of $\mu_{n}$ and $v_{n}$. For all such $n$ we obtain, as before,

$$
T(r, f) \leqq K(q) \delta^{-1} \hat{N}(r, 0) \leqq K(q) \delta^{-1} v_{n}\left(\log \left|a_{n}\right|\right)^{2}
$$

for $q^{1 / 2}\left|a_{n}\right| \leqq r \leqq q^{3 / 4}\left|a_{n}\right|$. We remark that this is exactly the same inequality as (3.3), the common idea being to obtain an inequality like (3.6) which expresses $T(R, f)$ in terms of $\hat{N}(R, 0)$.

We now let $\zeta_{1}, \ldots, \zeta_{M}\left(M=p_{n}\right)$ be the poles of $f(z)$ in the annulus $\left\{Q^{-1}\left|a_{n}\right| \leqq\right.$ $\left.|z| \leqq Q\left|a_{n}\right|\right\}$ with the above choice of $Q$ and set

$$
h(z)=f(z) \prod_{k=1}^{M}\left(z-\zeta_{k}\right) .
$$

If we now apply the Poisson-Jensen formula ([3], Theorem 1.1) to $h(z)$, for $|z|<$ $R=q^{1 / 2}\left|a_{n}\right|$, and ignore the contribution from the zeros we obtain

$$
\log |h(z)| \leqq m(R, h)+\Sigma^{*} \log \left|\frac{R^{2}-\bar{\zeta} z}{R(z-\zeta)}\right|,
$$


where $\Sigma^{*}$ denotes that the sum is taken over all poles $\zeta_{k}$ of $f(z)$ except those in the annulus $\left\{Q^{-1}\left|a_{n}\right| \leqq|z| \leqq Q\left|a_{n}\right|\right\}$. If $\left|z-a_{n}\right|=1$ then it is easily seen that, for our choice of $Q=\min \left(e^{1 / 2},(1+\delta / 4)^{-1} q^{1 / 2}\right)$ we have

for each $\zeta$ considered in $\Sigma^{*}$.

$$
\log \left|\frac{R^{2}-\bar{\zeta} z}{R(z-\zeta)}\right| \leqq K(q) \log \frac{2}{\delta}
$$

Hence, for $\left|z-a_{n}\right| \leqq 1$,

$$
\log |h(z)| \leqq K(q)\left\{T(R, f)+M \log \left|a_{n}\right|+n(R, \infty) \log \frac{2}{\delta}\right\} .
$$

But $n(R, \infty) \leqq K(q) T\left(q^{3 / 4}\left|a_{n}\right|, f\right)$ and so, for $\left|z-a_{n}\right| \leqq 1$,

$$
\begin{aligned}
\log |h(z)| & \leqq K(q) \log \frac{2}{\delta}\left\{T\left(q^{3 / 4}\left|a_{n}\right|, f\right)+p_{n} \log \left|a_{n}\right|\right\} \\
& \leqq K \delta^{-1} \log \frac{2}{\delta} v_{n}\left(\log \left|a_{n}\right|\right)^{2}(1+o(1)) \quad(n \rightarrow \infty) .
\end{aligned}
$$

The proof now follows in exactly the same manner as before. We define

$$
g(z)=f(z) \prod_{k=1}^{M}\left(z-\zeta_{k}\right) \prod_{j=1}^{N}\left(z-z_{j}\right)^{-1}=h(z) \prod_{j=1}^{N}\left(z-z_{j}\right)^{-1},
$$

where $z_{1}, \ldots, z_{N}\left(N=v_{n}\right)$ are the zeros of $f(z)$ in $D_{n}$. Since

$$
\log \left|\prod_{j=1}^{N}\left(z-z_{j}\right)^{-1}\right| \leqq v_{n} \log 2 \text { for }\left|z-a_{n}\right|=1,
$$

we obtain from the preceding estimate that, for $\left|z-a_{n}\right| \leqq 1$,

$$
\log |g(z)| \leqq K(q) \delta^{-1} \log \frac{2}{\delta} v_{n}\left(\log \left|a_{n}\right|\right)^{2}(1+o(1)) .
$$

Hence, for $\left|z-a_{n}\right| \leqq 1$

But,

$$
\log |f(z)| \leqq K \delta^{-1} \log \frac{2}{\delta} v_{n}\left(\log \left|a_{n}\right|\right)^{2}+\sum_{j=1}^{N} \log \left|z-z_{j}\right|-\sum_{k=1}^{M} \log \left|z-\zeta_{k}\right| .
$$

$$
\prod_{k=1}^{M}\left(z-\zeta_{k}\right)=\left(z-a_{n}\right)^{M}+\text { lower powers of }\left(z-a_{n}\right) .
$$

Hence for some $z_{0}$ on $\left|z-a_{n}\right|=\varrho_{n}$ we have

and so

$$
\left|\prod_{k=1}^{M}\left(z_{0}-\zeta_{k}\right)\right| \geqq \varrho_{n}^{M}
$$

$$
\log \left|f\left(z_{0}\right)\right| \leqq K(q) \delta^{-1} \log \frac{2}{\delta} v_{n}\left(\log \left|a_{n}\right|\right)^{2}+(N-M) \log \varrho_{n} .
$$


We now repeat the above argument with $f(z)-1$ instead of $f(z)$. Note that the poles of $f(z)$ and $f(z)-1$ are the same and hence we obtain, for the same point $z_{0}$,

$$
\log \left|f\left(z_{0}\right)-1\right| \leqq K(q) \delta^{-1} \log \frac{2}{\delta} v_{n}\left(\log \left|a_{n}\right|\right)^{2}+(N-M) \log \varrho_{n}
$$

since, by the Argument Principle and Lemma 1, $f(z)$ has the same number of zeros and 1-points in $D_{n}$ for all large $n$. As before, since $n \in E$, we have $N-M=v_{n}-p_{n}>$ $\delta v_{n} / 2$. But, either $\left|f\left(z_{0}\right)\right| \geqq 1 / 2$ or $\left|f\left(z_{0}\right)-1\right| \geqq 1 / 2$ or both and so we obtain

$$
\frac{\delta v_{n}}{2} \log \frac{1}{\varrho_{n}} \leqq K(q) \delta^{-1} \log \frac{2}{\delta} v_{n}\left(\log \left|a_{n}\right|\right)^{2}(1+o(1)) .
$$

This leads to estimate

$$
\log \frac{1}{\varrho_{n}} \leqq K(q) \delta^{-2} \log \frac{2}{\delta}\left(\log \left|a_{n}\right|\right)^{2}
$$

for all large $n$. Hence Theorem 4 is proved.

\section{Proof of Lemma 1}

We define, for $1<Q<q^{1 / 2}$, the set $A\left(Q, n_{0}\right)$ by

$$
A\left(Q, n_{0}\right)=\bigcup_{n=n_{0}}^{\infty}\left\{Q\left|a_{n}\right| \leqq|z| \leqq Q^{-1}\left|a_{n+1}\right|\right\} .
$$

If $f(z)$ satisfies the hypotheses of Theorem 4 then we show first that there is an integer $n_{0}$ and a constant $c_{0}>0$ such that $|f(z)| \geqq c_{0}$ in $A\left(Q, n_{0}\right)$. Since $\delta(\infty, f)>0$ there are, for all large $r$, points on the circle $|z|=r$ where $|f(z)|$ is large. Since $f(z) \neq 0,1, c$ in $A\left(Q, n_{0}\right)$ the function $F(z)=(1 / f(z)-1)(1 / c-1)^{-1}$ is analytic in these annuli and omits the values 0 and 1. The standard application of Schottky's Theorem ([1], p. 232) as before shows that there is a constant $K(Q)$ such that $|F(z)| \leqq K$ in $A\left(Q, n_{0}\right)$. For such $z$

and so

$$
\left|\frac{1}{f(z)}\right| \leqq 1+K\left|\frac{1}{c}-1\right|
$$

$$
|f(z)| \geqq c_{0}=\left(1+K\left|\frac{1}{c}-1\right|\right)^{-1} \text {. }
$$

We now show that $f(z) \rightarrow \infty$ as $z \rightarrow \infty$ through the set $\bigcup_{n-1}^{\infty}\left\{z:|z|=q^{1 / 2}\left|a_{n}\right|\right\}$ though it will be clear from the proof that we could replace this set by $A(Q, 1)$. As before $K$ denotes a generic constant depending on $Q$, and so on $q$, and not necessarily the same at each occurrence. We now select three numbers $q_{1}, q_{2}, q_{3}$ satisfying the following conditions 
a) $1<q_{i}<q^{1 / 2} \quad i=1,2,3$,

b) $\left(q_{1}-1\right)$ is very small,

c) $\left(q_{2}-1\right)$ is very small, but very large compared to $\left(q_{1}-1\right)$,

d) $q_{3}$ is large subject to the conditions $q_{1}+2 q_{3} \leqq 1+q^{1 / 4}, 2 q_{1}<q_{3}+1$.

We do not specify $q_{1}, q_{2}$ and $q_{3}$ though it will be clear in what follows how this is to be done.

Let us pick any point, $z_{0}$, say, on $\left\{|z|=q^{1 / 2}\left|a_{n}\right|\right\}$. The circle $\left\{\left|z-z_{0}\right|=\right.$ $\left.\left(q_{2}-1\right) q^{1 / 2}\left|a_{n}\right|\right\}$ is cut by the annulus $\left\{q_{1}^{-1} q^{1 / 2}\left|a_{n}\right| \leqq|z| \leqq q_{1} q^{1 / 2}\left|a_{n}\right|\right\}$ into two pieces. A point $z_{1}$ is chosen, in a manner to be specified later, lying on that arc $A_{1}$ of the circle which lies in the annulus in the counter-clockwise direction along $\left\{|z|=q^{1 / 2}\left|a_{n}\right|\right\}$ from $z_{0}$. We now consider the arcs of $\left\{\left|z-z_{i}\right|=\left(q_{2}-1\right) q^{1 / 2}\left|a_{n}\right|\right\}$ cut off by the same annulus and choose a point $z_{2}$ on the similar arc. Continuing in this way right round the narrow annulus we obtain a sequence $\left\{z_{0}, z_{1}, z_{2}, \ldots, z_{\alpha}\right\}$ of points, where $\alpha=\alpha(q)$ such that

e)

$$
\text { e) } \begin{gathered}
\left\{|z|=q^{1 / 2}\left|a_{n}\right|\right\} \subseteq \bigcup_{k=0}^{\alpha}\left\{\left|z-z_{k}\right|<(1 / 4)\left(q_{3}-1\right) q^{1 / 2}\left|a_{n}\right|\right\} \\
\text { f) } \quad\left\{q_{1}^{-1} q^{1 / 2}\left|a_{n}\right| \leqq|z| \leqq q_{1} q^{1 / 2}\left|a_{n}\right|\right\} \subseteq \bigcup_{k=0}^{\alpha}\left\{\left|z-z_{k}\right|<2\left(q_{3}-1\right) q^{1 / 2}\left|a_{n}\right|\right\} \\
\\
\qquad\left\{q^{1 / 4}\left|a_{n}\right| \leqq|z| \leqq q^{3 / 4}\left|a_{n}\right|\right\} .
\end{gathered}
$$

The condition f) can be fulfilled since $q_{1}$ and $q_{3}$ are related by d).

In what follows, $T(r, f, \zeta), N(r, f, \zeta)$ etc. will refer to the Nevanlinna functions with the origin replaced by the point $\zeta$ so that, for example,

$$
m(r, f, \zeta)=\frac{1}{2 \pi} \int_{0}^{2 \pi} \log +\left|f\left(\zeta+r e^{i \theta}\right)\right| d \theta .
$$

We suppose that $\left|f\left(z_{0}\right)\right| \leqq c_{1}$, where $\left|z_{0}\right|=q^{1 / 2}\left|a_{n}\right|$ and $n$ is large and show that this leads to a contradiction, so that $f(z) \rightarrow \infty$ as $z=q^{1 / 2}\left|a_{n}\right| e^{i \theta} \rightarrow \infty$. From Jensen's theorem we have

and hence

$$
\begin{gathered}
m\left(\left(q_{2}-1\right) q^{1 / 2}\left|a_{n}\right|, f, z_{0}\right)+N\left(\left(q_{2}-1\right) q^{1 / 2}\left|a_{n}\right|, f, z_{0}\right) \\
=\log \left|f\left(z_{0}\right)\right|+m\left(\left(q_{2}-1\right) q^{1 / 2}\left|a_{n}\right|, \frac{1}{f}, z_{0}\right),
\end{gathered}
$$

$$
m\left(\left(q_{2}-1\right) q^{1 / 2}\left|a_{n}\right|, f, z_{0}\right) \leqq \log +\frac{1}{c_{0}}+\log c_{1}=c_{2} \quad \text { say }
$$

since $|f(z)| \geqq c_{0}$ everywhere in the range concerned.

Now the arc $A_{1}$ discussed above has angular measure exceeding $\beta=\beta(q)$ say, so there is some point $z_{1}$ on $A_{1}$ such that

$$
\left|f\left(z_{1}\right)\right| \leqq \frac{2 \pi}{\beta} c_{2}
$$


We now repeat the argument with $z_{1}$ in place of $z_{0}$, to find $z_{2}$ and continue in this manner. Thus we have constructed a sequence $\left(z_{0}, z_{1}, \ldots, z_{\alpha}\right)$ of points such that

$$
\left|f\left(z_{k}\right)\right| \leqq B=B\left(c_{0}, c_{1}, q\right) \quad k=0,1, \ldots, \alpha .
$$

Let $\zeta_{1}, \zeta_{2}, \ldots, \zeta_{L}$ be the poles of $f(z)$ lying in the annulus $\left\{q_{3}^{-1} q^{1 / 2}\left|a_{n}\right| \leqq|z| \leqq\right.$ $\left.q_{3} q^{1 / 2}\left|a_{n}\right|\right\}$ and define

$$
g(z)=f(z) \prod_{k=1}^{L}\left(z-\zeta_{k}\right)
$$

so that $g(z)$ is analytic and non-zero in the annulus. Note that $L=L(n)$. Applying the Poisson integral representation for $g(z)$ in the disc $\left\{\left|z-z_{k}\right| \leqq(1 / 2)\left(q_{3}-1\right) q^{1 / 2}\left|a_{n}\right|\right\}$ we find that, for $\left|z-z_{k}\right| \leqq(1 / 4)\left(q_{3}-1\right) q^{1 / 2}\left|a_{n}\right|$,

$$
\log |g(z)| \leqq 3\left\{m\left(\frac{1}{2}\left(q_{3}-1\right) q^{1 / 2}\left|a_{n}\right|, f, z_{k}\right)+m\left(\frac{1}{2}\left(q_{3}-1\right) q^{1 / 2}\left|a_{n}\right|, I_{1}^{L}\left(z-\zeta_{k}\right), z_{k}\right)\right\} .
$$

But, since $f(z)$ has no zeros in the discs under consideration,

$$
\begin{aligned}
m\left(\frac{1}{2}\left(q_{3}-1\right) q^{1 / 2}\left|a_{n}\right|, f, z_{k}\right) & \leqq m\left(\frac{1}{2}\left(q_{3}-1\right) q^{1 / 2}\left|a_{n}\right|, \frac{1}{f}, z_{k}\right)+\log \left|f\left(z_{k}\right)\right| \\
& \leqq \log ^{+} \frac{1}{\left|c_{0}\right|}+\log B .
\end{aligned}
$$

Hence, from (4.1),

$$
\log |g(z)| \leqq 3\left[\log +\frac{1}{\left|c_{0}\right|}+\log B+L \log \left(2 q_{3} q^{1 / 2}\left|a_{n}\right|\right)\right]=K(q) L \log \left|a_{n}\right|,
$$

for $\left|z-z_{k}\right| \leqq(1 / 4)\left(q_{3}-1\right) q^{1 / 2}\left|a_{n}\right|$. Thus, from e) above, we obtain

$$
\log |g(z)| \leqq K(q) L \log \left|a_{n}\right| \quad\left(|z|=q^{1 / 2}\left|a_{n}\right|\right)
$$

and therefore, with $r=q^{1 / 2}\left|a_{n}\right|$,

$$
m(r, f) \leqq m(r, g)+m\left(r, \prod_{1}^{L}\left(z-\zeta_{k}\right)^{-1}\right) .
$$

We obtain from (4.2) and a straightforward application of Jensen's theorem that

$$
m(r, f) \leqq K(q) L \log \left|a_{n}\right| \quad\left(r=q^{1 / 2}\left|a_{n}\right|\right) .
$$

In particular, since $\delta(\infty, f)>0$ we obtain

$$
T(r, f) \leqq K \delta^{-1} L \log r \quad\left(r=q^{1 / 2}\left|a_{n}\right|\right),
$$

and since we are assuming that $f(z)$ is transcendental we conclude that $L=L(n) \rightarrow \infty$ as $n \rightarrow \infty$. It is from this that our contradiction follows. From $\mathrm{f}$ ) it follows that there is some $k_{0}, 0 \leqq k_{0} \leqq \alpha$, such that the disc $\left\{\left|z-z_{k_{0}}\right|<2\left(q_{3}-1\right) q^{1 / 2}\left|a_{n}\right|\right\}$ con- 
tains at least $L / \alpha$ poles of $f(z)$. By an application of Jensen's theorem as before we obtain, with $R=2\left(q_{3}-1\right) q^{5 / 8}\left|a_{n}\right|$,

Hence

$$
m\left(R, f, z_{k_{0}}\right)+N\left(R, f, z_{k_{0}}\right)=m\left(R, \frac{1}{f}, z_{k_{0}}\right)+\log \left|f\left(z_{k_{0}}\right)\right| .
$$

$$
m\left(R, \frac{1}{f}, z_{k_{0}}\right) \geqq \frac{L}{\alpha} \int_{R_{1}}^{R} \frac{d t}{t}-\log B=\frac{\log q}{8 \alpha} L-\log B,
$$

where $R_{1}=2\left(q_{3}-1\right) q^{1 / 2}\left|a_{n}\right|$. Thus there must be some point $w$ satisfying

$$
\left|w-z_{0}\right|=2\left(q_{3}-1\right) q^{5 / 8}\left|a_{n}\right| \text { for which } \log \left|\frac{1}{f(w)}\right| \geqq \frac{\log q}{8 \alpha} L-\log B .
$$

Since such a point $w$ must, in particular lie in the annulus $\left\{q^{1 / 8}\left|a_{n}\right| \leqq|z| \leqq q^{-1 / 8}\left|a_{n+1}\right|\right\}$ we see that

$$
c_{0} \leqq|f(\omega)| \leqq B \exp \left(-\frac{\log q}{8 \alpha} L\right),
$$

which is a contradiction if $n$ and hence $L$ is large enough. Thus the hypothesis $\left|f\left(z_{0}\right)\right| \leqq c_{1}$ is untenable for large $n$ and so $|f(z)| \rightarrow \infty$ as $z \rightarrow \infty\left(|z|=q^{1 / 2}\left|a_{n}\right|\right)$ as required.

\section{Concluding remarks}

We do not discuss Theorem 1 further since the matter is somewhat complicated and the results depend on the particular choice of the multiplicities involved. The difficulties evidently arise in our choice of the subsequences $(\sigma)$ in Case II and, though our sequences are universal in the sense that they work in all cases, we do not wish to imply (and do not believe) that they are optimal for every possible choice of zeros of $f(z)$.

The original version of this paper contained an example which we claimed showed that $K(q, \delta)$ of Theorem 2 behaved like $O\left(\delta^{-1}\right)$ as $\delta \rightarrow 0+$. However, we are indebted to Sakari Toppila who has pointed out to us that our argument is incorrect and that, for the functions we considered, $K(q, \delta)$ is independent of $\delta$. In addition Professor Toppila claims that, by using some of our above arguments in conjunction with a lemma of his, he can show that $K(q, \delta)$ is independent of $\delta$ in general. Moreover he has now dealt with the critical case of $K=1 / 2$ in Theorem 1 by showing that $K=1 / 2$ is not permissible in that theorem. 


\section{References}

[1] Baker, I. N., and L. S. O. Liverpool: Picard sets of entire functions. - Math. Z. 126, 1972, $230-238$.

[2] Baker, I. N., and L. S. O. Liverpool: Further results on Picard sets of entire functions. - Proc. London Math. Soc. (3) 26, 1973, 82-98.

[3] Hayman, W. K.: Meromorphic functions. - Clarendon Press, Oxford, 1964.

[4] Toppila, S.: Some remarks on the value distribution of entire functions. - Ann. Acad. Sci. Fenn. Ser. A I 421, 1968, 1-11.

[5] Valiron, G.: Sur les valeurs déficientes des fonctions algébroïdes méromorphes d'ordre nul. - J. Analyse Math. I, 1951, 28-42.

[6] Winkler, J.: Bericht über Picardmengen ganzer Funktionen. - Topics in analysis. Colloquium on Mathematical Analysis, Jyväskylä 1970. Edited by O. Lehto, I. S. Louhivaara and R. Nevanlinna. Lecture Notes in Mathematics 419, Springer-Verlag, Berlin-Heidelberg-New York, 1974, 384-392.

University College

Department of Mathematics

London W. C. 1

England

Received 13 August 1979
Imperial College

Department of Mathematics

London S. W. 7

England 